\title{
PROPUESTA PARA LA REHABILITACIÓN DE ÁREAS AFECTADAS POR MINERÍA DE FLUORITA. CASO DE ESTUDIO: ESTABLECIMIENTO CERROS NEGROS, CÓRDOBA, ARGENTINA
}

\author{
PROPOSAL FOR THE REHABILITATION OF AREAS AFFECTED BY FLUORITE MINING. \\ CASE STUDY: ESTABLECIMIENTO CERROS NEGROS, CÓRDOBA, ARGENTINA
}

\author{
Gabriela Andrea SACCHI ${ }^{1}$, Luis Ignacio JARA POZA ${ }^{1}$, Aldo Antonio BONALUMI², \\ Pablo HEREDIA ${ }^{3}$ \\ ${ }^{1}$ Facultad de Ciencias Exactas, Físicas y Naturales. Universidad Nacional de Córdoba. Escuela de Geología. Departamento de \\ Geología Básica. E-mails: gsacchi@unc.edu.ar; glijpoza@gmail.com \\ ${ }^{2}$ Facultad de Ciencias Exactas, Físicas y Naturales. Universidad Nacional de Córdoba. Secretaría de Minería de la Provincia de \\ Córdoba. E-mail: aldo.bonalumi@unc.edu.ar \\ ${ }^{3}$ Fluorita Córdoba SA. E-mail: produccioncrn@comininet.com.ar
}

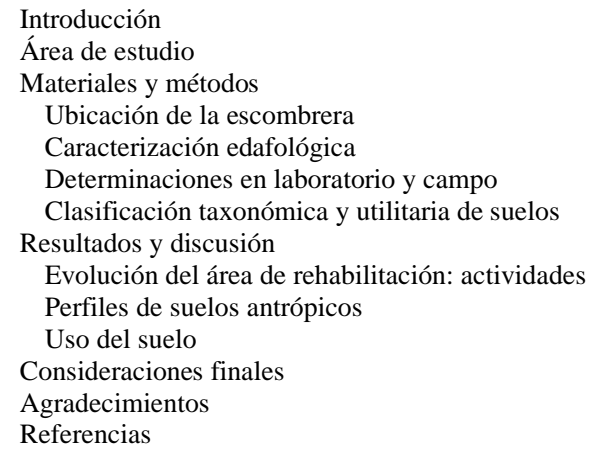

RESUMEN - La minería, es una actividad económica relacionada a la explotación y extracción de minerales, siendo responsable de profundas modificaciones en el medio natural. A través de ella, se obtienen la mayoría de los recursos necesarios para el desarrollo de la humanidad y el bienestar social del hombre. De esta forma, es necesario plantear el desarrollo de esta actividad humana pero de forma equilibrada, asegurando la protección y conservación del medio ambiente. La relación positiva entre la extracción de recursos geológicos y la protección ambiental es posible, generando una rehabilitación y utilización del medio asegurando una puesta en valor paisajística y económica del entorno. La transferencia de rocas estériles para el relleno de huecos y el tapado de frentes rocosos junto con la reconstrucción de suelos, permiten una rehabilitación del medio en sentido estricto. El propósito de este trabajo es contribuir al conocimiento de técnicas de rehabilitación de escombreras, en el caso de estudio en una explotación minera de fluorita, Establecimiento Cerros Negros, con la generación de suelos artificiales sustentables para el desarrollo de una actividad económica agrícola.

Palabras clave: agricultura, cantera, minería, rehabilitación, suelos.

ABSTRACT - Mining is an economic activity related to the exploration and production of minerals, being responsible for important changes in the natural environment. Through it, most of the resources needed for the development of humanity and social welfare of man are obtained. Thus, it is necessary to consider the development of this human activity but evenly, ensuring the protection and preservation of environment. The positive relationship between the extraction of geological resources and environmental protection is possible, generating environmental rehabilitation and ensuring use start economic landscape and environmental value. The transference of sterile rocks for filling gaps of rocky fronts together with the soil reconstruction, allow a rehabilitation means strictly. The purpose of this work is to contribute to the knowledge of techniques for rehabilitation of debris, in the case of study in a mining of fluorite, Establecimiento Cerros Negros, with the generation of sustainable artificial soils for the development of an agricultural economic activity.

Keywords: agriculture, mining, quarry, rehabilitation, soils.

\section{INTRODUCCIÓN}

La minería a cielo abierto es un tipo de disturbio antrópico que afecta todos los factores del ecosistema (ej. vegetación, fauna, suelos, aguas), las geoformas del terreno $y$ las condiciones microclimáticas (Montes de OcaRisco \& Mayda Lloa-Carcassés, 2013). El emplazamiento de toda actividad extractiva está condicionado tanto por la distribución natural como por la existencia de los recursos mineros, no existiendo la posibilidad de seleccionar más de un emplazamiento. De esta forma, es necesario un análisis integrado referido a la viabilidad económica y ambiental del proyecto minero en la única localización posible, que es aquella donde se ubica el yacimiento. Si bien estas actividades generan importantes beneficios 
a la sociedad también ocasionan importantes inconvenientes, siendo uno de los más nocivos la contaminación del medio ambiente (Actis, 2009). La degradación producida por esta actividad ha sido documentada globalmente, existiendo un sin número de referencias de los daños ambientales generados (Matos \& Martins, 2006; ArranzGonzález, 2011; Montes de Oca-Risco \& Mayda Lloa-Carcassés, 2013). En las décadas del 60 y 70 , se intentaba restablecer las condiciones originales del sitio degradado, actualmente se observa un pasaje del objetivo a buscar situaciones en las cuales la estabilidad del ambiente y su sustentabilidad sean efectivamente garantizadas, en particular, por medio de la instalación de un uso adecuado de suelo (Yazbek Bitar, 2002).

La conservación de los valores ecológicos, paisajísticos, productivos y científico-culturales de un lugar o territorio es una actitud activa. La conservación aun siendo necesaria, no es solamente suficiente, también es necesario mejorar, recuperar y rehabilitar aquellos recursos, ecosistemas o paisajes degradados para otros usos (Gómez Orea, 1994; Arranz- González \& Alberruche Del Campo, 2007). Entendiéndose por rehabilitación el proceso que tiende a establecer y suministrar protección para terrenos estables y seguros y de ecosistemas autosostenibles que deben ser compatibles con su ambiente natural, y adecuados para la utilización planeada de la tierra en el futuro. Debe ser considerada como parte central del negocio y debe ser integrada por completo dentro de los planes de operación de la cantera (Dietrich, 1990; WBCSD, 2011).

Las situaciones que generalmente se encuentran con posterioridad a la extracción minera abarcan el abandono de huecos y escombreras, con diferentes grados de impacto en el ambiente. La elaboración de suelos se plantea como una alternativa para la problemática actual en áreas mineras, donde se programa la gestión de residuos y la rehabilitación de áreas intensamente degradadas (ej. Arranz-González 2011; Uzarowicz \& Skiba, 2011). Estos suelos creados antrópicamente reciben el nombre de Tecnosoles, Antrosoles (IUSS, 2015), suelos mineros (Arranz-González, 2011) y suelos alterados y transportados por el hombre (USDA, 2014).

En este trabajo se propone investigar una solución para la rehabilitación de áreas modificadas por actividad minera con una generación importante de rocas estériles, asegurando una puesta en valor tanto paisajista como económico con el entorno. Estas actividades permitirán transformar sistemas degradados insustentables, en áreas económicamente sustentables, representadas por un sistema edáfico construido con capacidad de uso agrícola.

\section{ÁREA DE ESTUDIO}

El área de estudio corresponde a un sitio de rehabilitación de escombreras de la explotación minera de fluorita en el Establecimiento Cerros Negros, perteneciente a la empresa Fluorita Córdoba SA. Se encuentra ubicada hacia el oeste de la localidad Río de los Sauces, departamento Calamuchita, provincia de Córdoba. Entre las coordenadas 32³0'17,94"S - 6447'25,64"O y $32^{\circ} 30 ' 22,33^{\prime \prime S}$ - 64²7'20,84"O; a una altitud aproximada de $1200 \mathrm{msnm}$ (Figura 1).

Geológicamente se ubica en el entorno del batolito Cerro Áspero $\left(440 \mathrm{~km}^{2}\right)$ perteneciente a las Sierras Pampeanas de Córdoba (sierra de Comechingones). La característica estructural principal de este batolito es la conspicua circularidad de sus plutones y estructuras internas, desarrolladas completamente bajo condiciones de presión menor a $2 \mathrm{~Kb}$ (Pinotti et al., 2002). La litología del área, está integrada por rocas metamórficas (esquistos, gneises, migmatitas, anfibolitas y mármoles) y rocas ígneas de composición granítica del correspondiente batolito. El mismo está constituido por el emplazamiento sucesivo de tres plutones que individualmente desarrollan variaciones faciales (unidades internas, externas, cuspidales y cortejo de diques asociados). Las unidades internas conforman más del $60 \%$ de la superficie del batolito Cerro Áspero y están representadas por monzogranitos biotíticos de grano grueso a muy grueso, porfírícos. Su composición mineralógica está integrada por cuarzo, microclino, plagioclasa y biotita, con presencia variable de accesorios como muscovita, circón, apatito, fluorita, esfena, allanita, rutilo, ilmenita y magnetita. (Pinotti et al., 2002).

En las unidades externas, cuspidales y diques dominan leucogranitos de dos micas o exclusivamente muscovíticos, cuyas composiciones varían entre monzogranitos y granitos álcali feldespáticos. Gonzalez Chiozza 
(2004) estableció que en la formación de los depósitos minerales de este distrito Cerro Áspero se distinguieron cinco (5) estadios paragenéticos, siendo los intermedios los que presentan mayores contenidos de W y Mo y los más tardíos los más ricos en sulfuros con participación de $\mathrm{Bi}, \mathrm{Cu}, \mathrm{Zn}$, $\mathrm{Pb}$, Ag y $\mathrm{Au}$, entre otros. La wolframita es el mineral de mena más importante que se encuentra asociado con leyes que varían entre 1 y 1,6 \% en peso de $\mathrm{WO}_{3}$, con algunas excepciones de hasta 3 \%, (Brodtkorb et al., 2016). En este Distrito, el
Establecimiento Cerros Negros tiene su principal potencial en la concentración de fluorita y no está vinculado temporalmente con la mineralización metalífera.

Los depósitos se componen de fluorita, cuarzo, ópalo, calcedonia y pirita (Gonzalez Chiozza, 2004). Este batolito, es portador de las mayores concentraciones de fluorita de las Sierras Pampeanas, con una reserva total mayor de 1000000 th y leyes entre 40 y $60 \%$ de $\mathrm{CaF}_{2}$ (Coniglio, 2006).

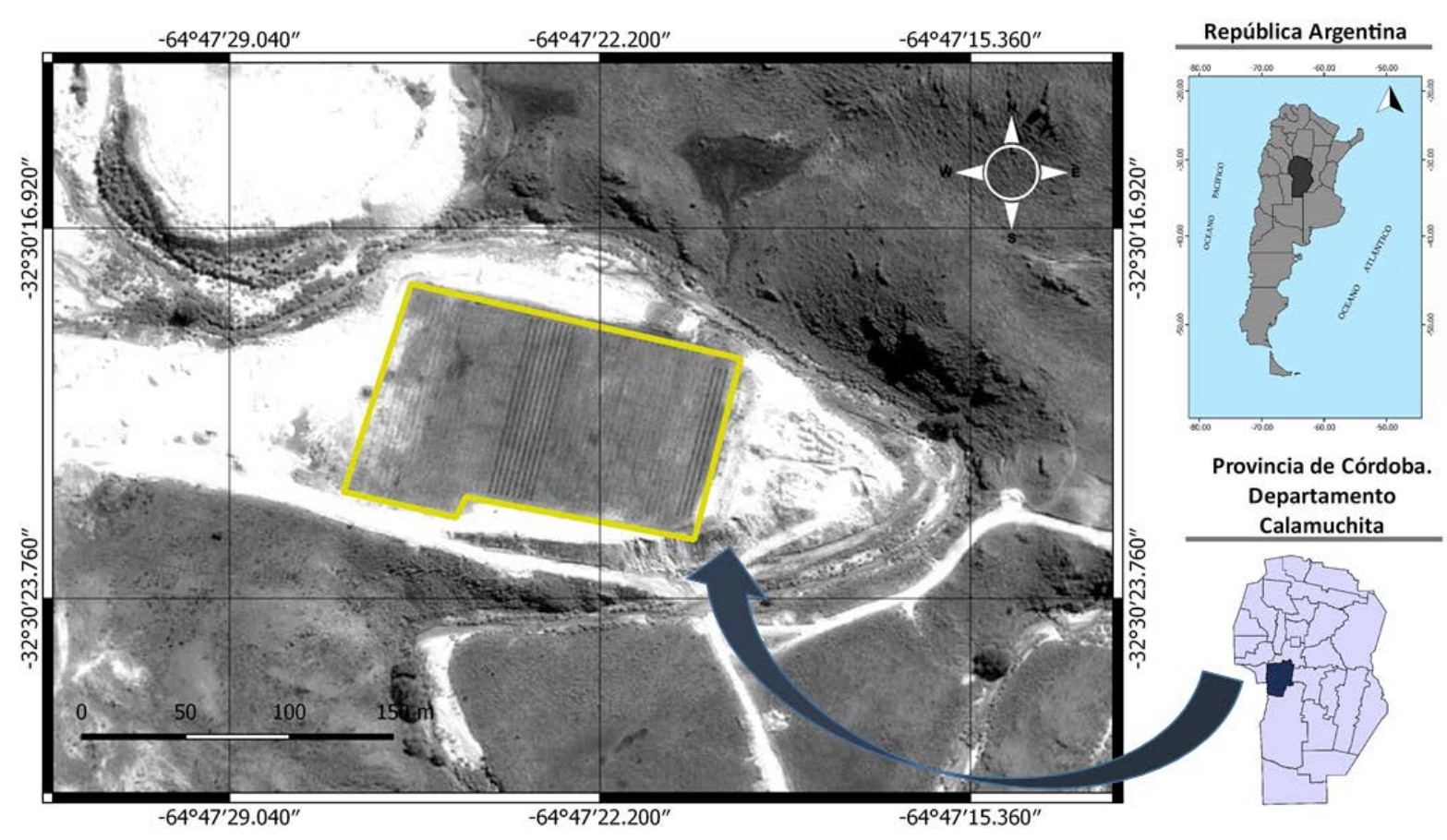

Figura 1 - Situación geográfica del área de estudio.

El régimen de precipitaciones del área de interés, es irregular, tipo monzónico, con un semestre lluvioso (octubre a marzo), que concentra el 81 - $82 \%$ del agua pluvial, y un semestre seco (abril a septiembre), con sólo 18 - $19 \%$ del agua precipitable (INTA \& MAG y RR, 1994). La alta dinámica del paisaje produce en general suelos jóvenes, de escaso desarrollo, someros $(<50 \mathrm{~cm})$, apoyados sobre materiales autóctonos (regolito). Los perfiles dominantes son A-R y A-Cr, con texturas franco arenosas, porcentajes de fragmentos gruesos $(\varnothing>2 \mathrm{~mm})$ mayores a $60 \%$ y pH 6. Corresponden al orden taxonómico Entisoles siendo clasificados como Ustorthentes líticos y paralíticos. En ambientes cóncavos de escaso gradiente, los suelos se han desarrollado a partir de materiales aluvionales de arrastre y los perfiles alcanzan mayor profundidad $(>50 \mathrm{~cm})$. El relieve escarpado, los suelos poco profundos, la baja retención de humedad, el drenaje excesivo y el grado de pedregosidad y rocosidad, determinan que el uso antrópico sea ganadero sobre pasturas naturales y/o forestales (Jarsún et al., 2006). El régimen de humedad de los suelos es ústico, es decir con un contenido de humedad limitada que se encuentra disponible cuando existen condiciones favorables para el crecimiento de las plantas. El régimen de temperatura es térmico, con una temperatura media anual $\geq$ de $15{ }^{\circ} \mathrm{C}$ pero $<$ de 22 ${ }^{\circ} \mathrm{C}$ y la diferencia entre la temperatura media del suelo en verano y en invierno es $>$ de $6{ }^{\circ} \mathrm{C}$ (INTA \& MAG y RR, 1994). La vegetación natural corresponde a un ecotono, con ejemplares tanto del Bosque serrano (nivel superior entre 800 y 1300 msnm) como del llamado Matorral Serrano, Arbustal de altura o "Romerillal" entre $~ 1300$ y 1700 msnm. Las especies dominantes son: Lithraea ternifolia, Fagara coco, Ruprechtia apetala, Festuca hieronymi, Stipa filiculmis y Stipa tenuissima, entre otras (Cabido 1994). La zona de estudio se encuentra ubicada en la cuenca alta del río Ctalamochita, puntualmente en las nacientes del 
río Quillinzo. El arroyo Los Vallecitos, afluente del mismo, sirve de abastecimiento de agua para las actividades económicas y domésticas que se desarrollan en la cantera.

Proyecto minero: en el establecimiento Cerros Negros, las vetas poseen una potencia entre 20 y 60 $\mathrm{cm}$, estando rellenas con fluoritas del estadio II, que corresponde a una fluorita finamente bandeada, crustiforme, con variaciones rítmicas de color, predominando el púrpura, blanco y violeta. La temperatura de formación varía entre 140 y $130{ }^{\circ} \mathrm{C}$ (Coniglio et al., 2010). Esta cantera es la única en Argentina que está en producción casi continua desde el año 1991, con un ritmo de 2500 ton/mes y leyes promedio de $32 \%$ de $\mathrm{CaF}_{2}$. En la actualidad este volumen de extracción es utilizado totalmente en la planta de flotación de la misma empresa. Esta planta concentradora produce una fluorita flotada con concentraciones entre 80 y $95 \%$ de $\mathrm{CaF}_{2}$. Las actividades de rehabilitación surgen conjuntamente con la explotación minera en sentido estricto, debido al gran volumen de rocas estériles obtenidas durante las actividades de producción, ya que la relación útil/estéril es de 1/13. Con esta relación los volúmenes de estériles alcanzan 32000 tn mensuales.

\section{MATERIALES Y MÉTODOS}

\section{Ubicación de la escombrera}

La construcción de la escombrera se orientó a $700 \mathrm{~m}$ hacia el este del actual frente de explotación. La disposición de estos materiales se realizó en un sector del establecimiento minero que carece de potencial productivo, con un diseño de geoforma acorde con el paisaje circundante.

La posición en el relieve corresponde a la media loma baja (backslope) - pie de loma (footslope), siendo la superficie de apoyo lateral las laderas del ambiente escarpado serrano cuyos gradientes de pendiente oscilan entre 25\% y 45 $\%$. La forma de la escombrera es típica de un terraplén formado a partir del vertido libre de material estéril sobre una superficie de baja pendiente $(<25 \%)$. Este material constituye el sustrato que es ocultado por una pantalla de suelos y cultivos (Figura 2). En el caso de estudio se utilizó solamente un recubrimiento edáfico, debido a que no se necesitaba un aislamiento completo de la escombrera, porque ésta no contenía materiales lixiviables nocivos. Los criterios para la conformación de la escombrera corresponden a los principios teóricos básicos establecidos en la bibliografía especializada (ej. Aramburu, 1990; ANEFA, 2007; Actis, 2009; CTAP, 2013).

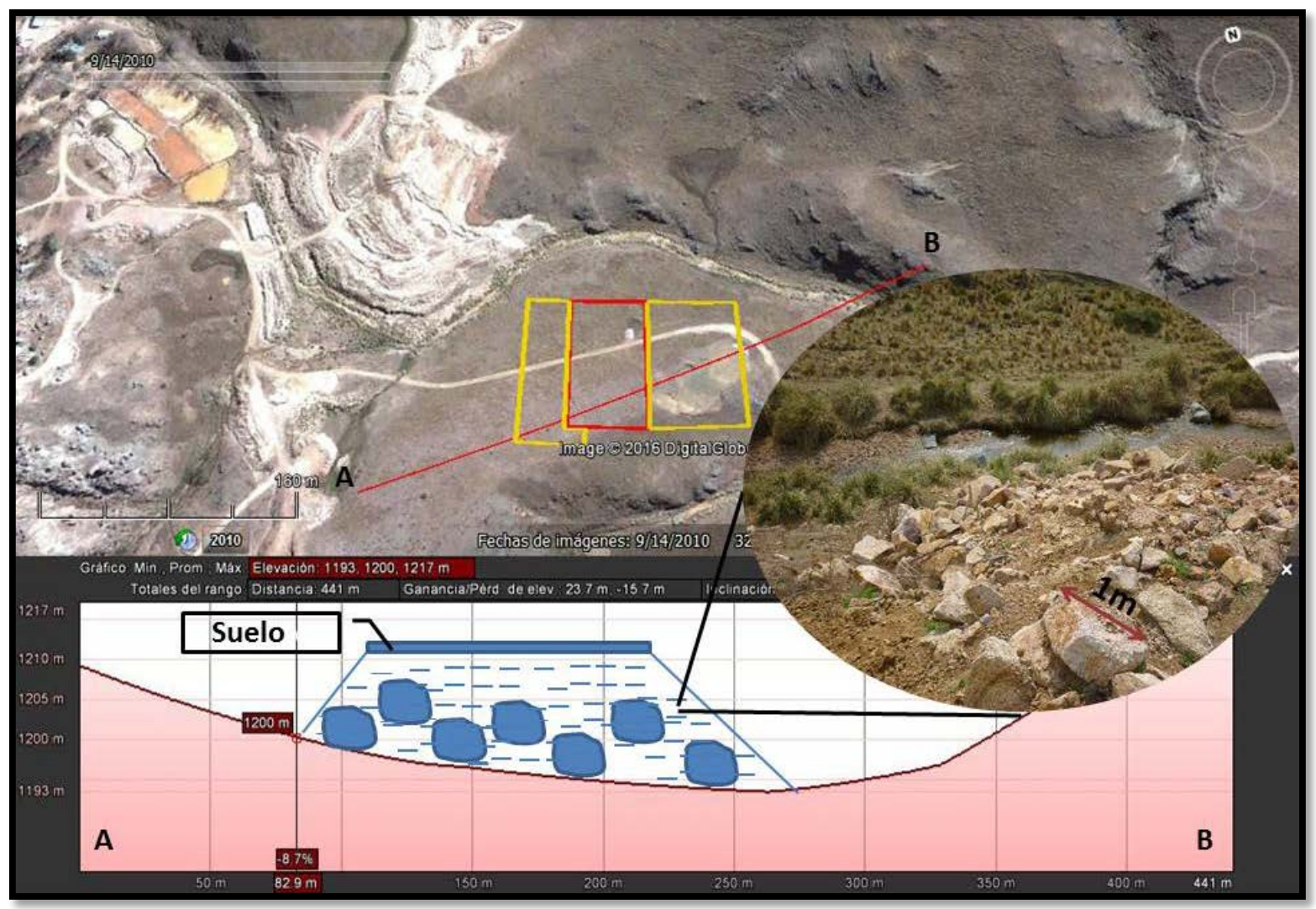

Figura 2 - Perfil esquemático mostrando la forma de la escombrera. 


\section{Caracterización edafológica}

Sobre la escombrera se crearon suelos con un potencial de uso agrícola. Para realizar su caracterización, se definieron dos (2) transectas perpendiculares de estudio, donde cada $5 \mathrm{~m}$ se realizaron observaciones de la profundidad efectiva de los perfiles y la textura del horizonte A. Las variaciones de estas propiedades permitieron la caracterización de tres (3) perfiles típicos, los cuales fueron descriptos de acuerdo a los criterios de Schoeneberger et al., (2012).

\section{Determinaciones en laboratorio y campo}

En las muestras de suelos de los distintos perfiles estudiados se determinó: carbono orgánico oxidable (Cox) por el método de Walkey \& Black (1934), pH (relación 1:2,5 suelo:agua), conductividad eléctrica (CE) (relación 1:2,5 suelo:agua), capacidad de intercambio catiónico (CIC) por extracción con solución de acetato de amonio $1 \mathrm{~mol} \mathrm{L-1}$ ajustado a pH 7,0 (Page et al., 1982), carbonato de calcio $\left(\mathrm{CaCO}_{3}\right)$ por el método del calcímetro e hidrógeno de cambio $\left(\mathrm{H}^{+}\right)$(Jackson, 1986). La saturación con bases (SB) se determinó a partir de la relación entre el valor S (suma de bases) y el $\mathrm{H}^{+}$. La granulometría por el método de pipeta de Robinson (Schlichting et al., 1995) y tamizado en húmedo luego de destruir materia orgánica con $\mathrm{H}_{2} \mathrm{O}_{2}$ y $\mathrm{CaCO}_{3}$ con $\mathrm{HCl}$ dispersando la muestra con $\mathrm{NaOH}$, separándose las siguientes fracciones: arcilla $(<2 \mu \mathrm{m})$, limos $(2-50 \mu \mathrm{m})$, arenas $(50-2000 \mu \mathrm{m})$ y fragmentos gruesos $(>$ $2000 \mu \mathrm{m})$. Los ensayos de infiltración en campo se realizaron de acuerdo a la metodología propuesta por USDA (1999).

\section{Clasificación taxonómica y utilitaria de suelos}

Los suelos fueron clasificados taxonómicamente de acuerdo al sistema americano Soil Taxonomy hasta la categoría de familia textural (USDA, 2014). La capacidad utilitaria de las tierras para uso agrícola (CU) se determinó de acuerdo al Sistema de Clasificación propuesto por el Servicio de Conservación de Suelos de los Estados Unidos (1977) con ligeras modificaciones establecidas por técnicos del Instituto Nacional de Tecnología Agropecuaria de Argentina (en Sacchi, 2013). Definiendo la clase y subclase de capacidad de uso.

\section{RESULTADOS Y DISCUSIÓN}

\section{Evolución del área de rehabilitación: actividades}

Las actividades mineras tanto a cielo abierto como subterráneas producen una gran cantidad de materiales de descarte que es necesario almacenar en lugares adecuados, planeando su ubicación en zonas económicas y en condiciones especiales en lo que se refiere a su estabilidad, seguridad e integración con el entorno. Las rocas estériles procedentes de la cobertura de las operaciones de obras, se depositan generalmente como fragmentos gruesos en pilas que constituyen las denominadas escombreras (Actis, 2009). El lugar de emplazamiento seleccionado para la ubicación de la escombrera permitió adecuar la topografía final a la existente, quedando como una superficie equiparable a una terraza alta del arroyo Los Vallecitos. Este cauce bordea la escombrera sobre la margen derecha, en un diseño de meandros encajados en el relieve serrano circundante. De esta forma el área de rehabilitación se comienza a diseñar a principios del año 2013, con la reconstrucción del terreno con la conformación de un terraplén, que presenta características poco adecuadas para el desarrollo de la vegetación, por la escasa presencia de materia orgánica y la baja proporción de elementos finos (Aramburu et al., 1990).

En una primera etapa, la superficie aproximada del área destinada a rellenar fue de media hectárea. De esta superficie se extrajeron unos 50 - $80 \mathrm{~cm}$ de suelo que se depositaron en un lugar de espera próximo al área a rehabilitar. Sobre la superficie libre de material edáfico se colocaron $\sim 65000 \mathrm{~m}^{3}$ de material estéril (granito de variada granulometría) proveniente de la cantera, conformando de esta manera la base del terraplén. Sobre esta base, se depositaron unos $9500 \mathrm{~m}^{3}$ del suelo previamente extraído. Este material se fue compactando con maquinaria pesada (ej. camiones y palas), a medida que el mismo se iba depositando, quedando una capa edáfica de aproximadamente $20 \mathrm{~cm}$ de profundidad. Con el fin de lograr una mezcla entre los dos sustratos depositados (estéril y suelo) y reducir la compactación, sobre la superficie se pasa un arado de disco con una profundidad de $\sim 20 \mathrm{~cm}$. Esta técnica de preparación del suelo artificial produce efectos favorables para el crecimiento de plantas ya que facilita el aumento del sistema radical, y además anula la escorrentía al aumentar la velocidad de infiltración, lo que permite la entrada de agua a 
capas profundas del suelo (CTAP, 2013). Por último se agregaron entre 30 y $40 \mathrm{~cm}$ más de suelo para aumentar su profundidad efectiva. A partir del año 2014 se anexa una hectárea más a la superficie original rehabilitada, donde la profundidad del sustrato suelo es mayor $(\sim 90$ $\mathrm{cm})$. De esta forma, queda conformado un Microrrasgo Antropogénico Constructivo, de acuerdo a características diagnósticas definidas para suelos alterados y transportados por el hombre (USDA, 2014).

Terminada la restitución morfológica definitiva del terreno, con una pendiente final menor a $1 \%$, el perímetro de la superficie rehabilitada de 110 x 110 m se delimitó con alambrado, para asegurar la estructura edáfica contra posible perturbación de animales de la zona. En la superficie edáfica estabilizada, se comenzaron a desarrollar actividades agrícolas. El acondicionamiento de los materiales para hacerlos más aptos para la revegetación es una etapa crucial en una rehabilitación de canteras (Paradelo, 2013). La mecánica de los ecosistemas en las primeras fases del procesos de restauración son críticas para alcanzar el éxito final del proyecto (Alarcón Martínez, 2017)

La secuencia temporal de las superficies construidas se muestra en la Figura 3.
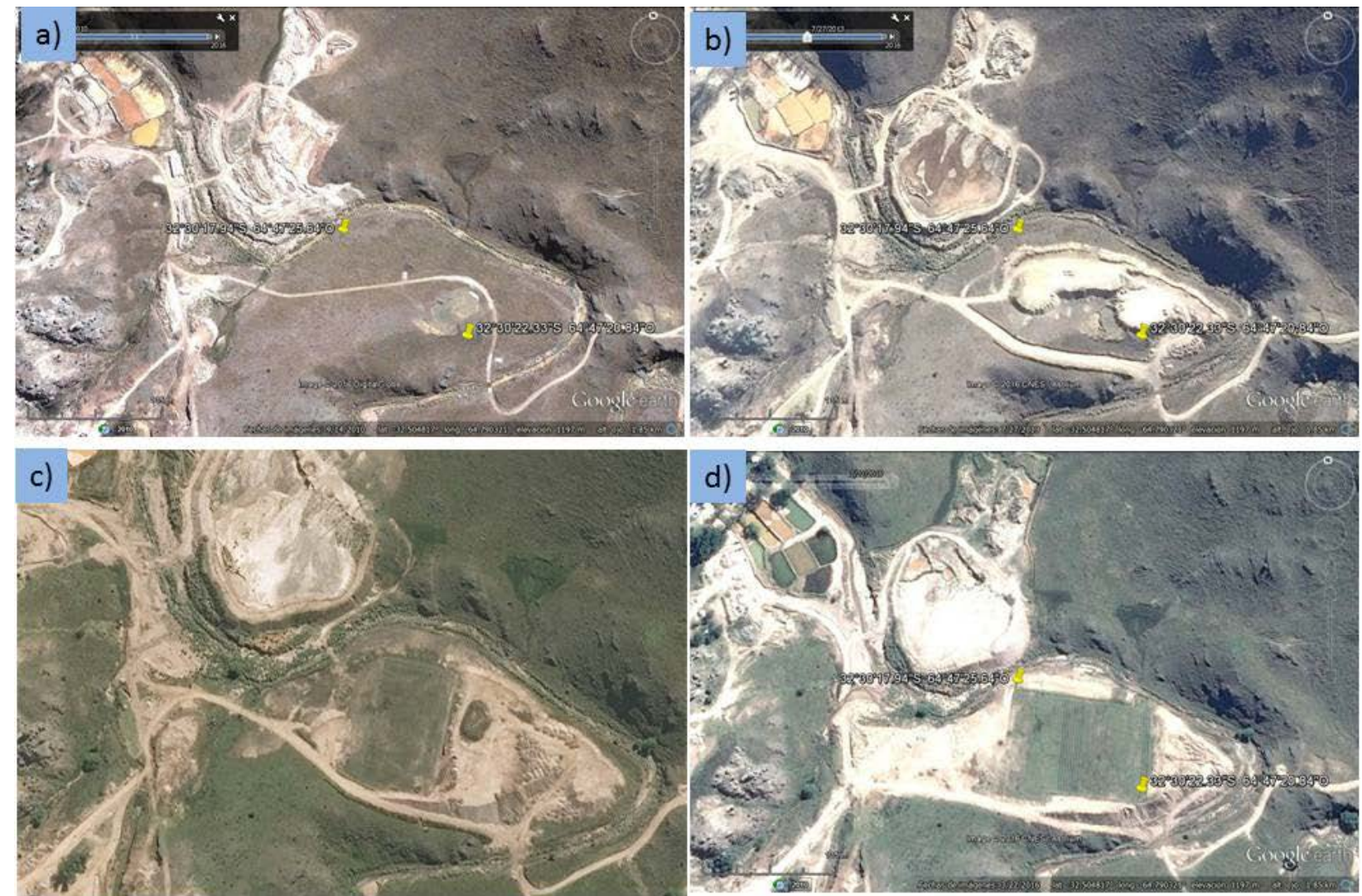

Figura 3 - Evolución de la escombrera a partir del año a) 2010; b) 2013; c) 2014; hasta d) 2016.

Las actividades de rehabilitación comprendieron solo un sector de la explotación minera, siendo realizadas en forma integrada con el proyecto de explotación, constituyendo por lo tanto una fase más del mismo. De esta forma, es posible recuperar las zonas degradadas al mismo tiempo que se llevan a cabo las actividades extractivas, minimizando el impacto ambiental y los costes económicos de la rehabilitación final del predio afectado por la actividad (ANEFA, 2007). El cierre final de la cantera depende de muchos factores, entre ellos reservas de flúor en vetas, situación socio-económica del país y precio internacional de la fluorita, no encontrándose a la fecha establecido.
La definición de la forma de rehabilitación y el destino final de un área de explotación no comprenden propuestas universalmente aplicables, dependiendo de las circunstancias concretas de cada explotación, las empresas y viabilidad de implantar una determinada actuación. Cada rehabilitación debe ser abordada en su contexto particular, sin ideas preconcebidas, para optar por aquella solución que permita satisfacer todas las condiciones de entorno que se apliquen en esas circunstancias concretas (ANEFA, 2007). En la actualidad, no se cuenta con una metodología de cierre de canteras, con carácter sistémico e integral, que sirva como guía a la hora de tomar decisiones São Paulo, UNESP, Geociências, v. 39, n. 3, p. 739 - 750, 2020 
futuras para el logro de la sustentabilidad (Guerrero-Almeida et al., 2014). Sin embargo, los procedimientos elaborados para la recuperación de las áreas afectadas por la minería deben permitir el mejoramiento de la calidad ambiental, social y económica en las zonas afectadas por la actividad y el logro de una minería responsable (Montes de Oca-Risco \& Mayda Lloa-Carcassés, 2013).

\section{Perfiles de suelos antrópicos}

El recubrimiento edáfico artificial creado, actúa como un sistema abierto y dinámico, donde se desarrollan los procesos elementales de génesis, al permitir el movimiento del agua a través del mismo. Esto favorece el cumplimiento de sus funciones vitales tal como se comportaría un cuerpo edáfico natural, al tener una función biológica, alimentaria y de soporte. Las principales características morfológicas y las propiedades físico químicas de los perfiles de suelos identificados en la cubierta de la escombrera se resumen en las tablas 1 y 2 respectivamente.

Estos suelos de naturaleza antrópica, en terminología anglosajona han sido denominados mine soils, es decir suelos mineros o de minas (Arranz-González, 2011). Severson \& Gough (1983) los definen como cualquier tipo de material de recubrimiento (topsoil, subsuelo, estéril o cualquier combinación de estos materiales) que queda intencionadamente en la superficie del terreno minero como medio de crecimiento de la vegetación. En los Grupos de Referencia de Suelos los mismos se clasifican como Technosoles “...siendo suelos cuyas propiedades y edafogénesis están dominadas por su origen técnico...” (IUSS, 2015).

En el caso de estudio, los suelos fueron creados en forma rigurosamente planificada mediante el extendido superficial de materiales geológicos no consolidados y capas de suelo (ej. Arranz-González, 2011; Leguédois et al., 2016). Se encuentran formados por una sucesión de capas con contrastes granulométricos, que por su origen antrópico es común que presenten una variabilidad en la distribución vertical de los horizontes en profundidad, siendo frecuente la presencia de límites abruptos con forma típica quebrados. Es decir, límites que en ciertas partes de su definición horizontal se encuentran interrumpidos (Figura 4, P2). Los límites irregulares que presentan concavidades más profundas que anchas, también son resultado de las actividades antrópicas de creación de suelos, producto de la labor de arado de disco (Figura 4, P1).

Las discontinuidades litológicas en los materiales de conformación de la escombrera, están indicadas utilizando números arábigos como prefijos, los mismos indican cambios significativos en la distribución del tamaño de partícula. Estas discontinuidades resultan de la superposición de los distintos materiales que conforman los perfiles, quedando bien diferenciados los pasivos ambientales en la base (sustrato) cubiertos por el material edáfico (topsoil). El límite entre estos dos materiales, escombrera y edáfico, condicionan la profundidad efectiva de los perfiles, la cual presenta una amplia variabilidad en el área de rehabilitación. Esta situación condiciona tanto la profundidad vertical de los perfiles como su distribución horizontal (espacial) en la misma.

En la nomenclatura de los horizontes de estos suelos antrópicos se utiliza el símbolo de “intercalación” $(\wedge)$. El mismo se coloca como un prefijo en la designación de horizontes mayores, para indicar capas minerales u orgánicas de material transportado por el hombre. Este material se ha movido horizontalmente sobre un pedón a partir de un área fuente que se localiza fuera del pedón, como resultado directo de la actividad humana, siendo usual con la ayuda de maquinaria (USDA, 2014).

En los perfiles estudiados solamente se reconocieron los horizontes principales $\mathrm{A}, \mathrm{C}$ y $\mathrm{R}$. Los horizontes superficiales A fueron identificados principalmente por la estructura edáfica de bloques subangulares y colores oscuros producto de la acumulación de materia orgánica humificada. El horizonte $\mathrm{C}$ corresponde a un material sin estructura, masivo o formado por roca meteorizada (ej. $2^{\wedge} \mathrm{Cr}$ ). El horizonte $\mathrm{R}$ define el material de escombrera, con una dificultad que excede a la categoría alta para su excavación (Figura 4).

La melanización es el único proceso pedogenético que ha actuado en forma importante. Este proceso de oscurecimiento responsable del origen de los horizontes superficiales (A), es resultado de la evolución de restos orgánicos frescos hacia formas complejas (humus), con participación de los microorganismos del suelo. La expresión morfológica del proceso queda reflejada en los colores oscuros del horizonte superficial con value y croma en húmedo $\leq 3$ (tabla 1 ) y mayores contenidos de Cox con respecto al horizonte inferior subyacente (tabla 2). 
Tabla 1 - Descripción morfológica de los suelos.

\begin{tabular}{|c|c|}
\hline Horizonte & Descripción Morfológica \\
\hline \multicolumn{2}{|r|}{ 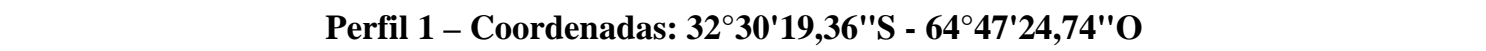 } \\
\hline$\wedge$ Ap & $\begin{array}{l}\text { 0-24 cm. color: en húmedo 10YR 3/1 (gris muy oscuro), en seco 10YR 3/2 (pardo grisáceo } \\
\text { muy oscuro); franco arenosa fina, < } 20 \% \text { fragmentos gruesos; bloques subangulares medios, } \\
\text { fuertes; friable; no plástico y ligeramente adhesivo; límite inferior claro ondulado. }\end{array}$ \\
\hline$\wedge \mathbf{A} 2 \mathbf{k}$ & $\begin{array}{l}\text { 24-36 cm. color: en húmedo } 10 \mathrm{YR} 3 / 2 \text { (pardo grisáceo muy oscuro), en seco } 10 \mathrm{YR} 3 / 3 \\
\text { (pardo oscuro); franco arenosa fina, < } 20 \% \text { fragmentos gruesos; bloques subangulares finos } \\
\text { a medios, moderados; friable; no plástico y ligeramente adhesivo; límite inferior abrupto } \\
\text { irregular. }\end{array}$ \\
\hline $2^{\wedge} \mathbf{R}$ & $\begin{array}{l}\text { 36-79 cm. color en húmedo 7,5 YR 6/6 (amarillo rojizo); roca firme; límite inferior abrupto } \\
\text { ondulado. }\end{array}$ \\
\hline $3^{\wedge} \mathrm{Cr}$ & $\begin{array}{l}79 \text { cm a más. color en húmedo 2,5 YR 5/6 (rojo); roca meteorizada con fragmentos de roca } \\
\text { inalterados }\end{array}$ \\
\hline
\end{tabular}

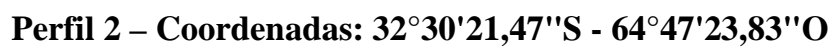

\begin{tabular}{c|l}
\hline$\wedge \mathbf{A p}$ & $\begin{array}{l}\text { 0-16 cm. color: en húmedo 10YR 2/1 (negro), en seco 10YR 3/3 (pardo oscuro); franco } \\
\text { arenosa fina, < 20\% fragmentos gruesos; bloques subangulares, medios y finos, débiles; } \\
\text { friable; no plástico, no adhesivo; límite inferior claro suave }\end{array}$ \\
\hline \multirow{A}{*}{$\mathbf{A} 2$} & $\begin{array}{l}\text { 16-35 cm. color: en húmedo 10YR 2/2 (pardo muy oscuro), en seco 10 YR 3/2 (pardo } \\
\text { grisáceo muy oscuro); franco gravilloso, < 30\% fragmentos gruesos; bloques subangulares } \\
\text { medios a gruesos moderados; friable; no plástico, no adhesivo; límite inferior abrupto } \\
\text { ondulado. }\end{array}$ \\
\hline $\mathbf{2} \mathbf{C r}$ & $\begin{array}{l}\text { 35-40 cm. color: en húmedo 10YR 4/3 (pardo oscuro a pardo), en seco 10YR 5/4 (pardo } \\
\text { amarillento); roca altamente meteorizada; suelto; límite inferior abrupto quebrado }\end{array}$ \\
\hline $\mathbf{3}^{\wedge} \mathbf{R}$ & $40-87 \mathrm{~cm}$. color en húmedo 2,5 YR 3/0 (gris muy oscuro); roca firme \\
\hline
\end{tabular}

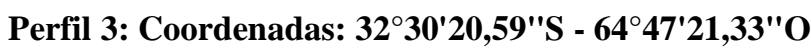

\begin{tabular}{c|l}
\hline$\wedge \mathbf{A p}$ & $\begin{array}{l}\text { 0-21 cm. color: en húmedo 10YR 3/2 (pardo grisáceo muy oscuro), en seco 10YR 3/3 (pardo } \\
\text { oscuro); franco arenosa, < 20\% fragmentos gruesos; estructura en bloques subangulares, } \\
\text { finos, débiles; friable; ligeramente plástico, no adhesivo; límite inferior claro suave }\end{array}$ \\
\hline \multirow{2}{*}{$\mathbf{A} \mathbf{A} 2$} & $\begin{array}{l}\text { 21-47 cm. color: en húmedo 10YR 2/2 pardo muy oscuro), en seco 10 YR 3/1 (gris muy } \\
\text { oscuro); franco arcillosa a franco gravilloso, > 30\% fragmentos gruesos; bloques } \\
\text { subangulares finos a medios, moderados, friable; plástico, ligeramente adhesivo; límite } \\
\text { inferior abrupto suave. }\end{array}$ \\
\hline $\mathbf{3}^{\wedge} \mathbf{C}$ & $\begin{array}{l}\text { 47-99 cm. color: en húmedo 10YR 3/3 (pardo oscuro), en seco 10YR 4/2 (pardo grisáceo } \\
\text { oscuro); arenosa franco grueso gravilloso, > 30\% fragmentos gruesos; masivo; friable; no } \\
\text { plástico, no adhesivo. }\end{array}$ \\
\hline $\mathbf{4} \wedge \mathbf{R}$ & $99 \mathrm{~cm}$ a más. Color en húmedo 2,5 YR 3/0 (gris muy oscuro); roca firme \\
\hline
\end{tabular}

Un horizonte A se puede desarrollar en estos suelos mineros en un plazo de aproximadamente cinco (5) años (Haering et al., 1993; Varela et al., 1993). El otro proceso es la gleización, definido principalmente por el escurrimiento lento, que permite un mayor tiempo de permanencia del agua en el perfil, saturando el suelo y provocando una ligera hidromorfía. La pendiente de la escombrera menor a $1 \%$ favorece la actuación de este proceso pedogenético. En el caso de estos suelos creados antrópicamente el factor de formación predominante es el tiempo, siendo suelos recientes y jóvenes (ej. Lehmann, 2006; Certini \& Scalenghe, 2011).

$$
\text { Los suelos fueron clasificados }
$$
taxonómicamente como Haplustoll anthroportico (Perfil 1 y 2) y Haplustoll anthrodensico (Perfil 3). Pertenecen al orden Molisoll, por la presencia de un epipedón mólico con las siguientes características definitorias: profundidad del horizonte superficial mayor a $18 \mathrm{~cm}$; contenido de Cox mayor a 0,6 \%; textura más fina que areno franco muy fina; value y croma $\leq 3$ (húmedo) y value $\leq 5$ (seco) y SB $>$ de $50 \%$ (tablas 1 y 2$)$. El 
calificativo anthroportico hace referencia a suelos que se formaron en materiales transportados por el hombre con $50 \mathrm{~cm}$ o más de espesor, mientras que el término anthrodensico indica que tienen un contacto dénsico debido a una compactación mecánica provocada por el uso de maquinaria pesada dentro de los $100 \mathrm{~cm}$ de la superficie del suelo mineral.

Tabla 2 - Propiedades físicas y químicas de los suelos.

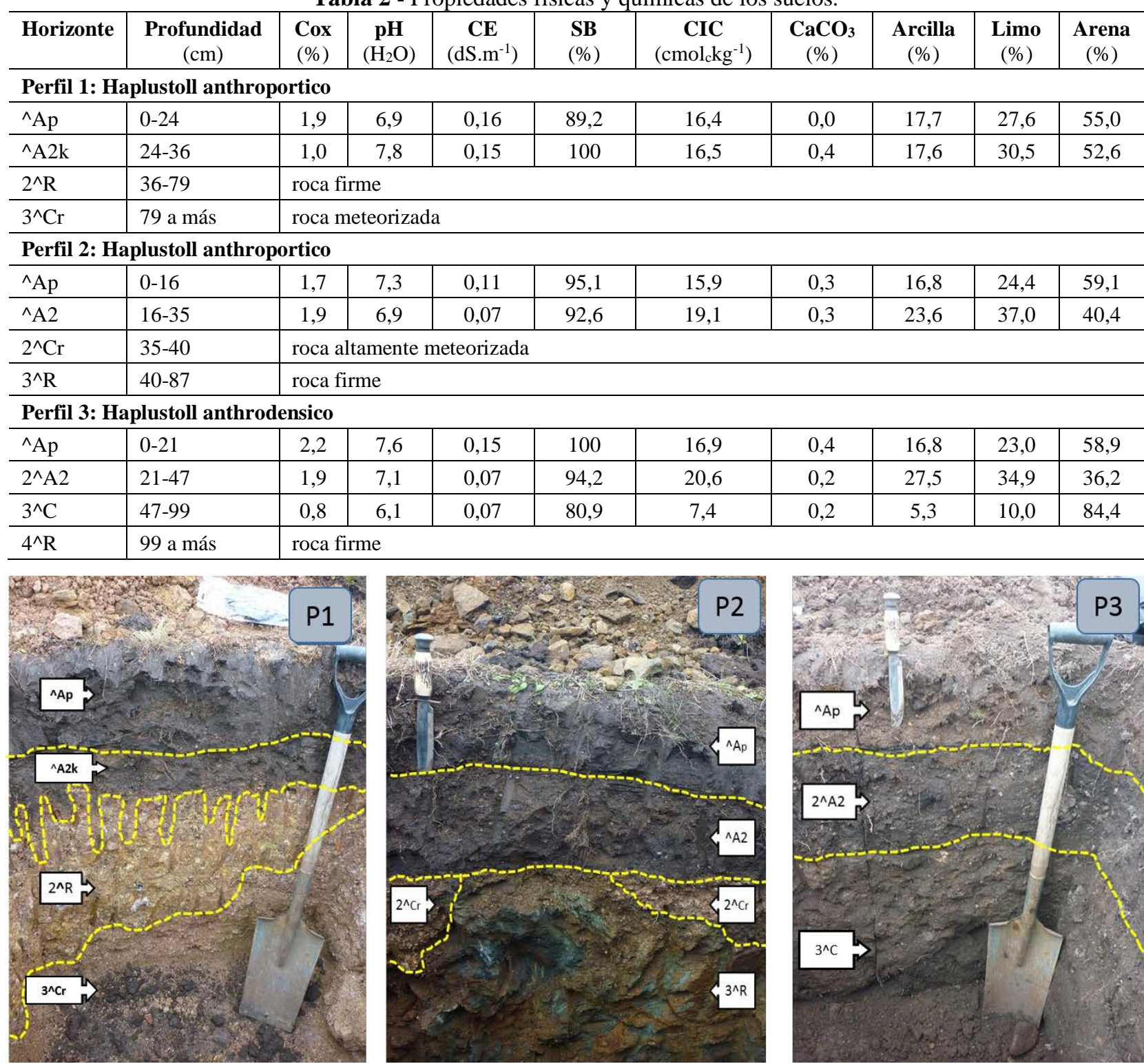

Figura 4 - Secuencias de horizontes y capas en los perfiles edáficos descriptos. Perfil 1 (P1), Perfil 2 (P2), Perfil 3 (P3).

En los horizontes superficiales la presencia de fragmentos gruesos en porcentajes menores al $20 \%$, permite diferenciar los suelos de los diferentes ambientes en la clasificación taxonómica a nivel de la categoría Orden. De esta manera, en el sistema serrano son clasificados como Entisoles y en el sistema rehabilitado como Mollisoles (tabla 1). La influencia antrópica queda evidenciada en la clasificación utilizada de los suelos a nivel de subgrupo.

\section{Uso del suelo}

Los suelos, en cuanto a la capacidad de uso fueron clasificados como agrícolas, siendo clase III (Perfil 3) y IV (Perfiles 1 y 2). Las limitaciones al uso se refieren a exceso de agua (w) condicionado por la profundidad efectiva (s), este impedimento físico que corresponde a un contacto duro principalmente dentro del metro de profundidad, favorece que el agua permanezca en el perfil por más tiempo. Los valores de infiltración resultaron entre $0,73 \mathrm{~cm} / \mathrm{h}$ y 9,4 $\mathrm{cm} / \mathrm{h}$, definiendo condiciones entre muy lento $\mathrm{y}$ moderado a medida que aumentaba la profundidad específica de los suelos. Esta condición se encontró favorecida también por el relieve subnormal del área de rehabilitación y las texturas relativamente finas de los horizontes. El clima (c) es un factor natural condicionante para 
el uso de los suelos, el régimen ústico define la existencia de meses con déficit hídrico. Debido a esta situación, se plantea el manejo de suelos con un sistema de riego a goteo, la capacidad es de 2L/h. La toma de agua para esta actividad se sitúo en el arroyo Los Vallecitos. Esta tecnología, más que una estrategia productiva orientada a maximizar la producción, es una herramienta para la planificación de la campaña agrícola, ya que gracias al riego es posible elegir con precisión la fecha de siembra y obtener estabilidad en los rendimientos de los cultivos. Además, se asegura la posibilidad de mantener los cultivos en invierno, cuando son más escasas las lluvias (Riera y Pereira, 2013).

En cuanto a las características químicas, los contenidos de Cox mayores a 0,6 \%, pHs entre ligeramente ácidos $(6,1)$ a moderadamente básicos $(7,8)$, valores de CE menores a $2 \mathrm{dSm}^{-1}$ (suelos no salinos) y alta SB (> 80\%), los suelos pueden definirse con condiciones óptimas para el sustento de cultivos (tabla 2). En estos suelos artificiales se colocaron plantines de vides de variedades tales como Cabernet, Sauvignon, Malbec, Syrah, Tempranillo, y Chardonnay entre otras. De esta forma se intenta determinar la adaptación al medio de cada cepa, continuando luego las actividades agrícolas con la plantación de la cepa que mejor se adapte a las condiciones ambientales del medio (Figura 5).

Las actividades de rehabilitación se completarán con la revegetación de los taludes de la escombrera, para tal fin se considera la siembra especies del ambiente natural tales como Stipas sp y Cortaderia argentea. Se ha podido observar

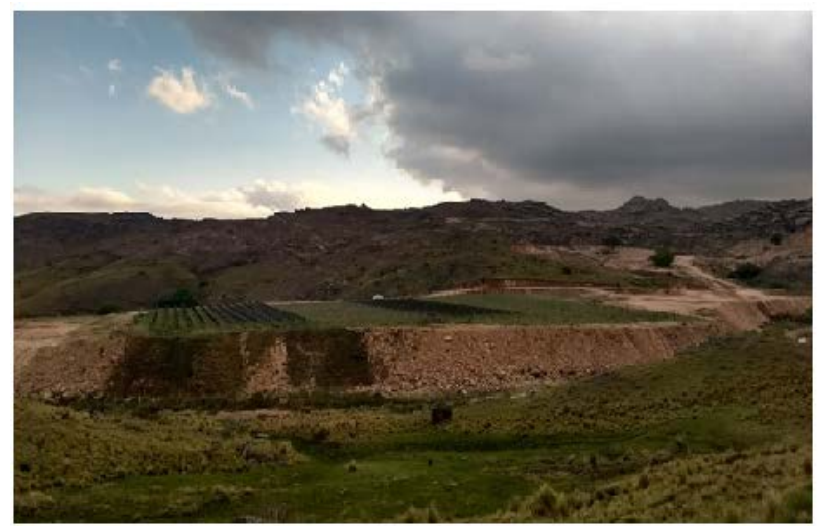

que el banco de semillas que tienen estos suelos artificiales es muy grande, habiendo germinado en el sustrato edáfico en forma natural innumerables especies entre las líneas de vides, alcanzando una cobertura de casi el 100\% (Figura 5). La presencia de esta cobertura superficial disminuye el riesgo de erosión de los suelos y favorece la conservación de la humedad.

En contraposición, los suelos que se extienden en el ambiente natural de las Sierras Pampeanas son de eminente aptitud forestal, ganadero con pasturas naturales y/o conservación de la fauna y flora natural. La capacidad de uso es no agrícola con clases VII/VIII, con limitaciones por problemas de erosión hídrica (e) y de suelo (s) definidas por texturas gruesas y profundidades efectivas menores a $50 \mathrm{~cm}$. Estas propiedades edáficas físicas junto con los gradientes de pendiente abruptos con grados que superan el 45 $\%$ y la presencia de afloramientos rocosos mayores al $20 \%$ condicionan la existencia de suelos poco desarrollados.

Comparando los sistemas naturales y los creados antrópicamente, se puede concluir que las actividades de rehabilitación permitieron minimizar la superficie afectada por la actividad minera realizando una restitución de los terrenos a otros usos, reutilizando los pasivos ambientales generados en dicha actividad (estériles). El impacto de esta actividad se logra minimizar debido a la baja potencialidad original que presentan los suelos en las áreas naturales de sierras (clases VII/VIII), permitiendo la rehabilitación crear suelos con una condición sustentable en sistemas cultivados (clases III/IV).

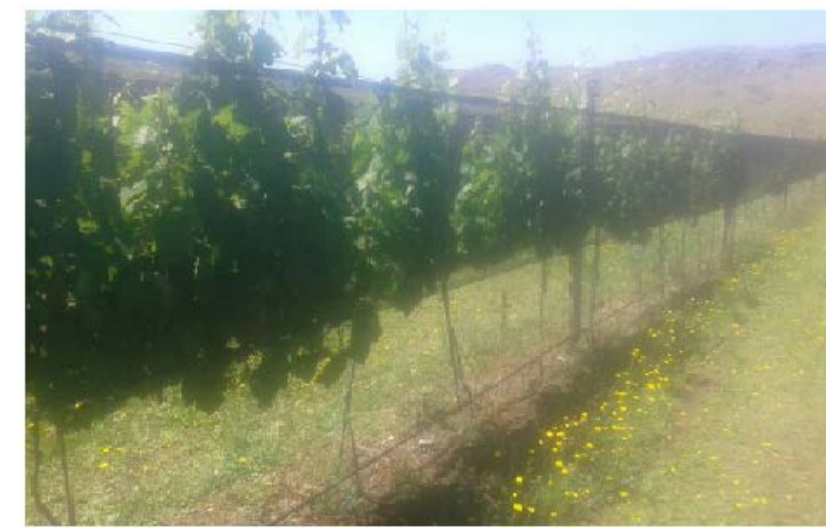

Figura 5 - Actividades agrícolas en suelos antrópicos del ambiente de escombrera.

\section{CONSIDERACIONES FINALES}

La rehabilitación de áreas mineras creando suelos productivos, cuyas características principalmente físicas son mejores que aquellas de los sistemas edáficos originales, constituye un

desafío actual para el uso sustentable de recursos naturales degradados. Esta práctica permite ordenar los pasivos ambientales generados en la actividad minera en una escombrera, que 
corresponde a una superficie con relieve subnormal, la cual actúa como el sustento de una cubierta edáfica con capacidad de uso agrícola. Como práctica se plantea la simultaneidad de tareas de explotación - rehabilitación en el predio afectado por la actividad minera, minimizando el impacto ambiental y económico al plantear una actividad sustentable en el tiempo. De esta forma, se formularon suelos artificiales que difieren de sus sistemas naturales del ambiente de sierra en la profundidad efectiva, texturas con menores contenidos de arena y fragmentos gruesos $(\varnothing$ < $2 \mathrm{~mm}$ ), favoreciendo las condiciones de infiltración y retención de agua en los perfiles. Estos suelos en el área de rehabilitación son jóvenes, con un desarrollo incipiente, formados por una sucesión de capas con contrastes granulométricos.

El desarrollo de los mismos, se puede considerar que comienza a partir de la estabilidad del relleno de escombrera. El único proceso pedogenético que ha actuado es la melanización, evidenciado por la presencia de un horizonte superficial mólico, que permite su clasificación taxonómica como Mollisoles. Los procesos pedogenéticos son similares a los que han actuado en los sistemas edáficos naturales. Sin embargo la modificación del relieve que condiciona la presencia de una superficie geomorfológica más estable junto con las características del material parental, ambos factores logrados por la intervención antrópica favorecerían una evolución de los suelos más rápida en el tiempo.

\section{AGRADECIMIENTOS}

Los autores agradecen a la empresa Fluorita Córdoba SA, en especial al Ing. Agr. Gonzalo Martínez por hacer posible la realización de este estudio, permitiendo el ingreso al sitio de estudio Establecimiento Cerros Negros y por el apoyo logístico brindado.

\section{REFERENCIAS}

ACTIS, R. Escombreras. Ubicación, estabilidad y contaminación ambiental. LDM Editorial, Argentina, 56 p., 2009.

ALARCÓN MARTINEZ, V. ¿Es posible restaurar medioambientalmente un suelo en una cantera de roca ornamental con residuos? Disp. en: http://www.conama local2017.conama.org/web/generico.php?idpaginas=\&lang=es \&menu=290\&id=77\&op=view. Acceso en: 18 de mayo de 2018.

ANEFA - ASOCIACIÓN NACIONAL DE EMPRESARIOS FABRICANTES DE ÁRIDOS. Manual de restauración de explotaciones mineras a cielo abierto de Aragón. Zaragoza, 135 p., 2007.

ARAMBURU, M.; ESCRIBANO, M.; DE FRUTOS, M. Restauración de zonas naturales alteradas por actividades mineras a cielo abierto. Informes de la Construcción, v. 42, n. 407, p 23-31, 1990.

ARRANZ-GONZÁLEZ, J. \& ALBERRUCHE DEL CAMPO, E. Minería, medio ambiente y gestión del territorio. Red DESIR (Desarrollo Sostenible - Ingeniería - Recursos Minerales). Universidad Politécnica de Madrid (UPM). ETSI Minas, Departamento de Ingeniería Geológica, Madrid, 95 p., 2007.

ARRANZ-GONZÁLEZ, J. Suelos mineros asociados a la minería de carbón a cielo abierto en España: una revisión. Boletín Geológico y Minero, v. 122, n. 2, p. 171-186, 2011.

BRODTKORB, M.; CONIGLIO, J.; MIRÓ, R. Los yacimientos metalíferos y la metalogenia de las Sierras Pampeanas de Córdoba. En: XIX CONGRESO GEOLÓGICO ARGENTINO, Córdoba, 1, 2014, p. 1-51. Relatorio de la Geología...Córdoba, 2014, p. 1-51.

CABIDO, M.; MANZUR, A.; CARRANZA, L.; GONZÁLEZALBARRACIN, C. La vegetación y el medio físico del Chaco Árido en la provincia de Córdoba, Argentina Central. Phytocoenologia, v. 24, p. 423-460, 1994.

CERTINI, G. \& SCALENGHE, R. Anthropogenic soils are the golden spikes for the Anthropocene. The Holocene, v. 21, p. 1269-1274, 2011.

CONIGLIO, J. Evolución petrológica y metalogenética del batolito Cerro Áspero en relación con el ciclo geoquímico endógeno del flúor, Sierra de Comechingones, Córdoba, Argentina, 2006. 163 p. Tesis (Doctoral) - Universidad Nacional de Río Cuarto, Río Cuarto.

CONIGLIO, J.; D’ERAMO, F.; PINOTTI, L.; DEMARTIS, M.; AGULLEIRO INSÚA, L.; PETRELLI, H. Control estructural de las mineralizaciones de fluorita del Batolito Cerro Áspero, Sierras Pampeanas de Córdoba. Revista de la Asociación Geológica Argentina, v. 67, n. 4, p. 507-520, 2010.

CTAP - CENTRO TECNOLÓGICO ANDALUZ DE LA PIEDRA. 2013. Estado del arte y criterios para la restauración de canteras en Andalucía y Marruecos Oriental. Disp. en: http://nubiaconsultores.es/wpcontent/uploads/2013/07/Gu\%C3\%ADa-de-restauracion-decanteras_ESP.pdf. Acceso en: 6 de abril de 2018.

DIETRICH, N. European rehabilitation projects reflect cultural and regional diversity. Rock Products, v. 93, n. 2, p. 45-47, 1990.

GÓMEZ OREA, D. Ordenación del Territorio. Una aproximación desde el medio físico. Publicaciones Instituto Geológico y Minero de España, Madrid, 238 p., 1994.

GONZÁLEZ CHIOZZA, S. Geología y metalogenia del Distrito Minero Cerro Áspero, Sierras Pampeanas de Córdoba, Argentina, 2004. 205 p. Tesis (Doctoral) Universidad Nacional de Buenos Aires, Buenos Aires.

GUERRERO-ALMEIDA, D.; CHACÓN-PÉREZ, Y.; FONSECA-HERNÁNDEZ, D.; COURT-POTRILLÉ, M. Metodología para la ejecución de un cierre de minas sustentable. Minería y Geología, v. 30, n. 3, p. 85-103, 2014.

HAERING, K.; DANIELS, W.; ROBERTS, J. Changes in mine soil properties resulting from overburden weathering. Journal of Environmental Quality, v. 22, p. 194-200, 1993.

INTA (INSTITUTO NACIONAL DE TECNOLOGÍA AGROPECUARIA) \& MAG y RR (MINISTERIO AGRICULTURA GANADERÍA Y RECURSOS RENOVABLES). Carta de Suelos Hoja 3366-12 Rio de Los Sauces - Hoja 3366-18 Alpa Corral. Córdoba. Córdoba, 97 p., 1994.

IUSS - INTERNATIONAL SOIL CLASSIFICATION SYSTEM FOR NAMING SOILS AND CREATING LEGENDS FOR

São Paulo, UNESP, Geociências, v. 39, n. 3, p. 739 - 750, 2020 
SOIL MAPS. World reference base for soil resources 2014 update 2015. World Soil Resources Reports $\mathbf{N}^{\circ}$ 106. Rome: FAO, 192 p., 2015.

JACKSON, M. Análisis Químico de suelos. Omega, Barcelona, 662 p., 1986.

JARSÚN, B.; GORGAS, J.; ZAMORA, E.; BOSNERO, H.; LOVERA, E.; RAVELO, A.; TASSILE, J. Recursos Naturales de la provincia de Córdoba, Los suelos nivel de reconocimiento 1:500.000. Agencia Córdoba Ambiente Instituto Nacional de Tecnología Agropecuaria. Córdoba, Argentina, 611 p., 2006.

LEGUÉDOIS, S.; SÉRÉ, G.; AUCLERC, A.; CORTET, J.; HUOT, H.; OUVRARD S.; WATTEAU, F.; SCHWARTZ, C.; MOREL, J. Modelling pedogenesis of Technosols. Geoderma, v. 262, p. 199-212, 2016.

LEHMANN, A. Technosols and other proposals on urban soils for the WRB (World Reference Base for Soil Resources). International Agrophysics, v. 20, p. 129-134, 2006.

MATOS, J. \& MARTINS, L. Reabilitação ambiental de áreas mineiras do sector português da Faixa Piritosa Ibérica: estado da arte e prespectivas futuras. Boletín Geológico y Minero, v. 117, n. 2, p. 289-304, 2006.

MONTES DE OCA-RISCO, A. \& MAYDA ULLOACARCASSÉS, M. Recuperación de áreas dañadas por la minería en la Cantera Los Guaos, Santiago de Cuba, Cuba. Revista Luna Azul, v. 37, p. 74-88, 2013.

PAGE, A.; MILLAR, R.; KENEY, D. Methods of soil analisys. Part 2. En: Agronomy Monograph 9.2. Madison, Wisconsin, American Society of Agronomy - Soil Science Society of America, p. 149-157, 1982.

PARADELO, R. Utilización de materiales compostados en la rehabilitación potencial de espacios afectados por residuos mineros y suelos de mina. Boletín Geológico y Minero, v. 124, n. 3, p. 405-419, 2013.

PINOTTI, L.; CONIGLIO, J.; ESPARZA, A.; D’ERAMO, F.; LLAMBÍAS, E. Nearly circular plutons emplaced by stoping at shallow crustal levels. Cerro Aspero batholith, Sierras Pampeanas de Córdoba, Argentina. Journal of South American Earth Sciences, v. 15, n. 2, p. 251-265, 2002.

RIERA, C. \& PEREIRA, S. Entre el riesgo climático y las transformaciones productivas: la agricultura bajo riego como forma de adaptación en Río Segundo, Córdoba, Argentina. Investigaciones Geográficas, Boletín del Instituto de Geografía, UNAM, v. 82, p. 52-65, 2013.

SACCHI, G. Guía de Trabajos prácticos. Cátedra de Cartografía y Conservación de Suelos. Informe inédito. Facultad Ciencias Exactas Físicas y Naturales, Universidad Nacional de Córdoba, Argentina, p. 53, 2013,

SCHLICHTING, E.; BLUME, H.; STAHR, K. Bodenkundliches Praktikum. Blackwell Wissenschafs Verlag, Berlín, 295 p., 1995.
SCHOENEBERGER, P.; WYSOCKI, D.; BENHAM, E. Soil Survey Staff. Disp. en: https://www.nrcs.usda.gov/Internet/ FSE_DOCUMENTS/nrcs142p2_052523.pdf. Acceso en: 3 de julio de 2017.

SERVICIO DE CONSERVACIÓN DE SUELOS, DEPARTAMENTO DE AGRICULTURA DE LOS ESTADOS UNIDOS. Manual de Conservación de Suelos. Limusa, México, 332 p., 1977.

SEVERSON, R. \& GOUGH, L. Rehabilitation Materials from Surface-Coal Mines in Western U.S.A. I: Chemical Characteristics of Spoil and Replaced Cover-Soil. Reclamation and Revegetation Research, v. 2, p. 83-102, 1983.

USDA - UNITED STATES DEPARTMENT OF AGRICULTURE. 1999. Guía para la Evaluación de la Calidad y Salud del Suelo. Departamento de Agricultura Servicio de Investigación Agrícola - Servicio de Conservación de Recursos Naturales - Instituto de Calidad de Suelos, 82 p., 1999.

USDA - UNITED STATES DEPARTMENT OF AGRICULTURE. Keys to Soil Taxonomy. Twelfth Edition. USDA - Natural Resources Conservation Service. Washington DC, 359 p., 2014.

UZAROWICZ, L. \& SKIBA, S. Technogenic soils developed on mine spoils containing iron sulphides: Mineral transformations as an indicator of pedogénesis. Geoderma, v. 163, p. 95-108, 2011.

VARELA, C.; VÁZQUEZ, C.; GONZÁLEZ-SANGREGORIO, M.; LEIRÓS, M.; GIL SOTRÉS, F. Chemical and physical properties of opencast lignite mine soils. Soil Science, v. 156, p. 193-204, 1993.

WALKEY, A. \& BLACK, I. An examination of Degtjareff method for determining soil organic matter and a proposed modification of the chromic acid titration method. Soil Science, v. 37, p. 29-38, 1934.

WBCSD - World Business Council for Sustainable Development. Disp. en: http://www.wbcsdcement.org/ pdf/CSI\%20Guidelines\%20on\%20Quarry\%20Rehabilitation\% 20(English)_Dec\%202011.pdf. Acceso en: 3 de julio de 2017.

YAZBEK BITAR, O. Recuperación de áreas degradadas por la minería en regiones urbanas. En: Repetto, F. y Karez, C. (Editores), II Curso Internacional de Aspectos Geológicos de Protección Ambiental. Oficina Regional de Ciencia de la Unesco, Montevideo, p. 332-345, 2002.

Submetido em 18 de maio de 2018 Aceito para publicação em 12 de agosto de 2020 